\title{
Investigation of Two-Dimensional Interaction between Piles due to Pile Driving Action
}

\author{
S. Sanchayan, U.G.A. Puswewala, S. Aravinthan, M. G. Kapilanath
}

\begin{abstract}
Pile driving displaces adjacent piles out of their position. This effect is simulated numerically in this work by using the finite element analysis package PLAXIS. For simplicity, the analysis is done under plane strain conditions and linear elastic material behaviour, which in fact converts the pile into infinitely long walls. Pile driving is modeled by progressive expansion of a cavity. The smear zone is modeled around the pile using material of lower stiffness to take into account the compression of soil around the pile. A parametric study is carried out to investigate the predicted lateral displacement of the head of a pile due to driving of an adjacent pile, by varying the stiffness of the soil and spacing between piles.
\end{abstract}

Keywords: Pile driving, FEM, Lateral displacement

\section{Introduction}

Pile foundations have been historically used to overcome the problems of soft soils. Piles are installed into the ground either by driving or boring a shaft and casting in-situ. It is reported that installation of a pile leads to interaction between afore installed adjacent piles (Terzaghi et al [1]). Especially in the case of pile driving, the adjacent piles can be displaced out of their original position.

In this work, the effects caused by pile driving on a pre-installed adjacent pile are investigated by using the finite element method (FEM), which is a versatile tool available to geotechnical engineers to solve complex soil structure interactions problems.

Two-dimensional finite element analysis was employed as the primary method of investigation. Finite element programme PLAXIS V8.2 [2] developed in Netherlands and widely used in the field of geotechnical engineering was used for the modelling.

Both pile and soil were modelled as linearly elastic materials, represented by Young's modulii and Poisson's ratios. The investigation was limited to two friction piles. One pile was considered as pre-installed while the other is driven into the ground and the disturbance of the pre-installed pile caused by the pile driving action was investigated by means of lateral displacement at the head of the adjacent pile. Friction pile is considered as the interaction is more critical in the case than the end bearing piles, because end bearing can offer some resistance and thus can limit the interaction caused by pile driving.
However, due to the inability of the software to handle three dimensional domain, the problem was modelled in two-dimensional domain. Therefore, the modelled problem in fact represents the interaction between two parallel, infinitely long walls.

A parametric study was carried out by varying the soil properties and spacing between piles, to investigate the effects of Young's moduli and spacing between piles on the lateral displacements at the head of the adjacent pile as caused by pile driving. Results of the study are presented in the ensuing sections.

\section{Idealisation}

The real problem involves a threedimensional half-space which is infinitely continuous domain except at the ground surface level, consisting of non-homogeneous, non-linear and antistrophic material possibly with a water table. Before carrying out any analysis, the problem should be properly idealised in order to be represented by the finite element model, which involves geometric idealization and material idealization.

S. Sanchayan, BSc Eng. (Moratuwa)

Eng. (Prof.) U. G. A. Puswewala, BSc Eng. (Moratuwa),

MEng. (AIT), PhD (Manitoba), CEng, MIE (Sri Lanka),

Professor of Civil Engineering, Department of Civil

Engineering, University of Moratuwa.

S. Aravinthan, BSc Eng. (Moratuwa)

M. G. Kapilanath, BSc Eng. (Moratuwa) 


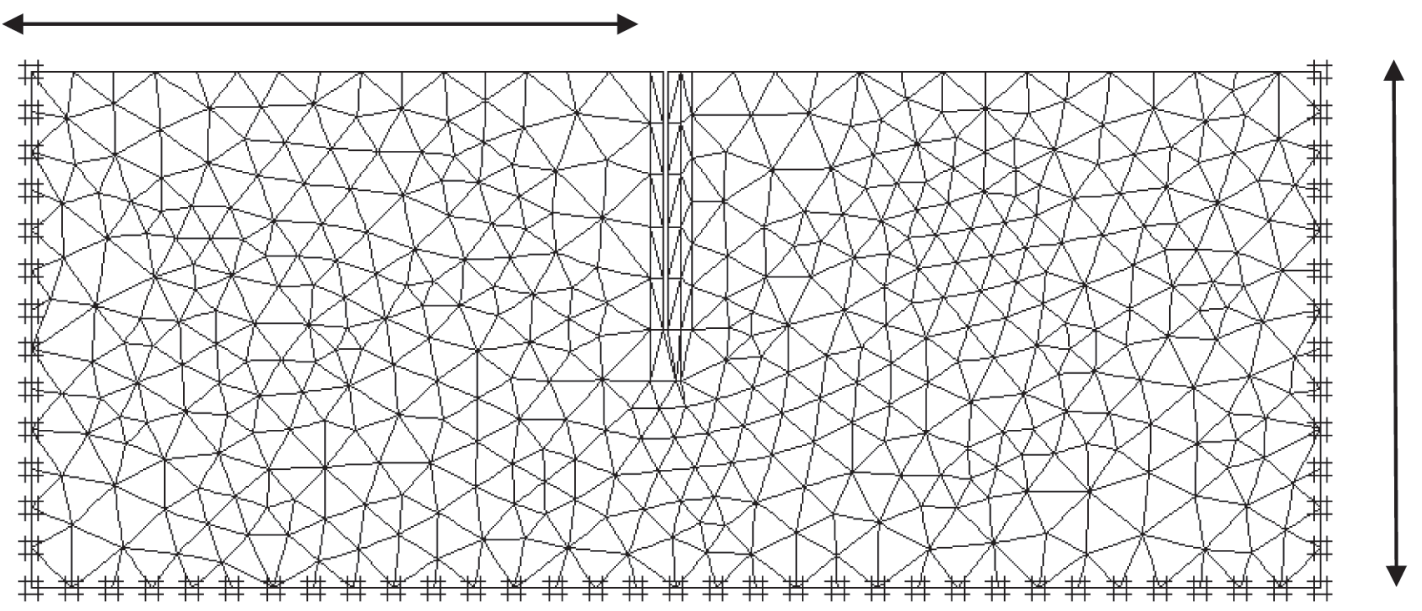

Figure 1 - Finite Element Mesh

Due to the limitations of the software version used in the analysis, three-dimensional spaces had to be idealised into two-dimensional spaces. For this purpose, plane strain conditions were used, which considers the strain in the direction perpendicular to the plane as zero. Thus a pile modelled under this idealization is in fact an indefinitely long wall.

Continuous domain cannot be modelled in the software. It should be approximated with an element mesh of finite extend. For that purpose the far boundary should be decided. Extend of the far boundary was determined in such a way that it has minimum influence on the numerical results. Displacements at the far boundary were fully fixed as it falls beyond the field of influence.

Selected far boundary was at 50d from the side of the piles and $\ell$ bellow the tip of the piles, where $\mathrm{d}$ is the diameter of the pile and $\ell$ is the length of the pile. The appropriately idealised finite element domain used in the research is shown in Figure. 1.

Soil and pile materials were idealised as homogeneous, isotropic and linear elastic material. The influence of the water table was not considered. The domain was assumed of consisting single material. Drained and undrained conditions were differentiated by the value of the Poisson's ratio. The model is considered as a weightless system since the study only investigates external effects.

Due to the idealizations and the numerical approach used in FEM, results obtained from the analysis are not exact but are practically reasonable. Any theoretical refinement only consumes more time and money, and therefore such refinement is not considered here for the sake of practicality.

\section{Finite Element Modelling}

PLAXIS has two triangular elements to model two dimensional problems, 6 node and 15 node elements. 15-noded triangular elements were with fine meshing in the entire domain as the numerical accuracy of the 15 nodal element is higher compared to the other element.

Clay was considered as the soil since piles are normally driven into weak soils. Undrained conditions were assumed since the pore water pressures do not stabilize in the short term, due to the low permeability of clays. Thus the Poisson's ratio of the clays was kept as 0.49 , which represents a nearly incompressible material.

Young's moduli for range of soils considered in the project are given in Table 1.The pile material was considered as concrete and was represented by a Young's modulus of $270000 \mathrm{kN} / \mathrm{m}^{2}$ and a Poisson's ratio of 0.2 . The diameter $(\mathrm{d})$ of the pile was chosen to be $0.4 \mathrm{~m}$ and the length $(\ell)$ was $10 \mathrm{~m}$.

Particular problem encountered in the modelling was to simulate the pile driving action in two dimensional domain especially with linear elastic constitutive model. Such simulation is complex considering all the nonlinearity caused by the action (Massout and Tassoulas [3]). A simple approach was adopted to simulate the pile driving in this work, and is described below in detail.

Pile driving causes compaction of the soil around the pile. As a results there exists a zone around the pile where the material in the zone behaves non- linearly. However, in this work the zone was using modelled linear elastic parameters. The purpose of modelling it in this work is to simulate the compaction caused by the pile driving which is otherwise impossible with the linear elastic idealisation 
which was achieved using a lower Young's modulus and Poisson's ratio for the zone.

The extent of the compacted area which is called the smeared zone is disputed by different researchers (Poulus [4]). However, USACE ([5]) identifies the region as $1 \mathrm{~d}$ around the pile perimeter for clayey soils and $2 \mathrm{~d}$ for sandy soils, where $d$ is the pile diameter. These values are considered in the current work.

Pile driving action was simulated using expansion of a cavity commencing from a negligible volume to the volume of the pile in five consecutive stages. The compaction effect caused by the pile driving action was taken into account by modelling the smeared zone around the cavity up to $1 \mathrm{~d}$ distance from the surface of the pile and to a sufficient distance (6.5d) below the tip of the pile, with a material of lower
Young's modulus and Poisson's ratio. The region representing the smeared or compacted region was also advanced in five stages as the cavity was expanded and advanced in five stages as illustrated by Figures 2 and 3. The shaded area in the figures represents the smeared zone. The horizontal arrow heads in Figure 3 indicate the direction at which the cavity is expanded. Note the size of the arrows is exaggerated in the figure. The numbers shown on the figures are node numbers used by the software and have no significant physical meaning.

The Young's modulus and the Poisson's ratio of the smeared region were arbitrarily selected as $100 \mathrm{kN} / \mathrm{m}^{2}$ and 0.1 . Investigations were carried out for different Young's modulus and Poisson's ratios of the smeared zone to check the sensitivity of these parameters on the results.

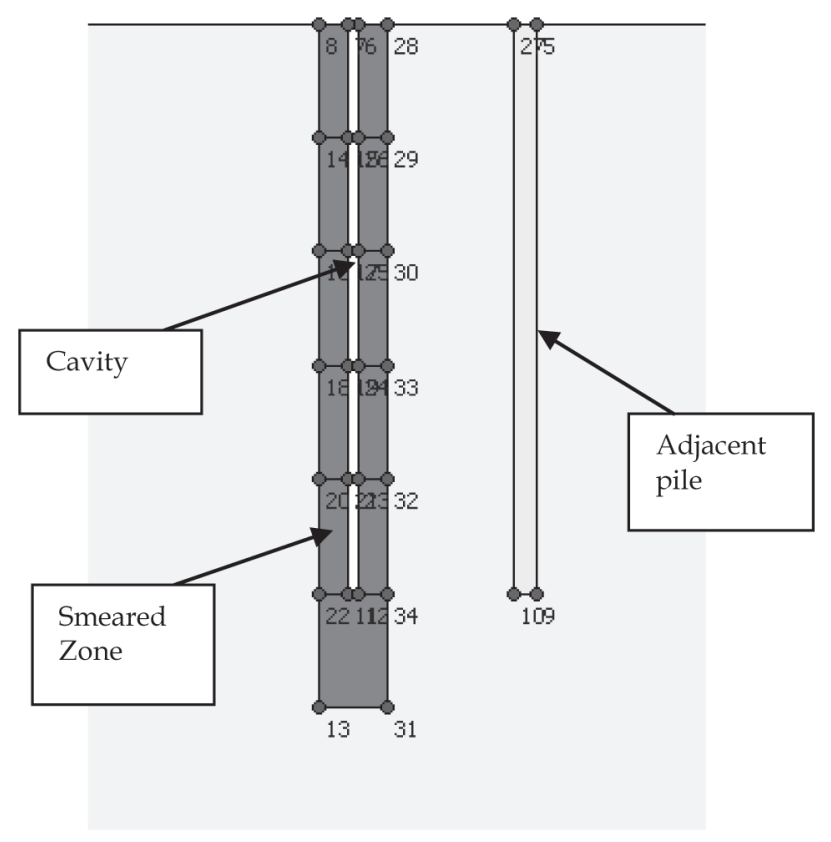

Figure 2 - Finite Element model used to simulate pile driving 
Table 1 - Range of values of Young's modulus for selected soils (after Bowles, 1977 [6])

\begin{tabular}{|l|l|}
\hline Type of Soils & Young's Modulus $\left(\mathrm{kN} / \mathrm{m}^{2}\right)$ \\
\hline Very soft clay & $300-3000$ \\
\hline Soft clay & $2000-4000$ \\
\hline Medium Clay & $4500-9000$ \\
\hline Hard Clay & $7000-20000$ \\
\hline Sandy Clay & $300000-42500$ \\
\hline
\end{tabular}

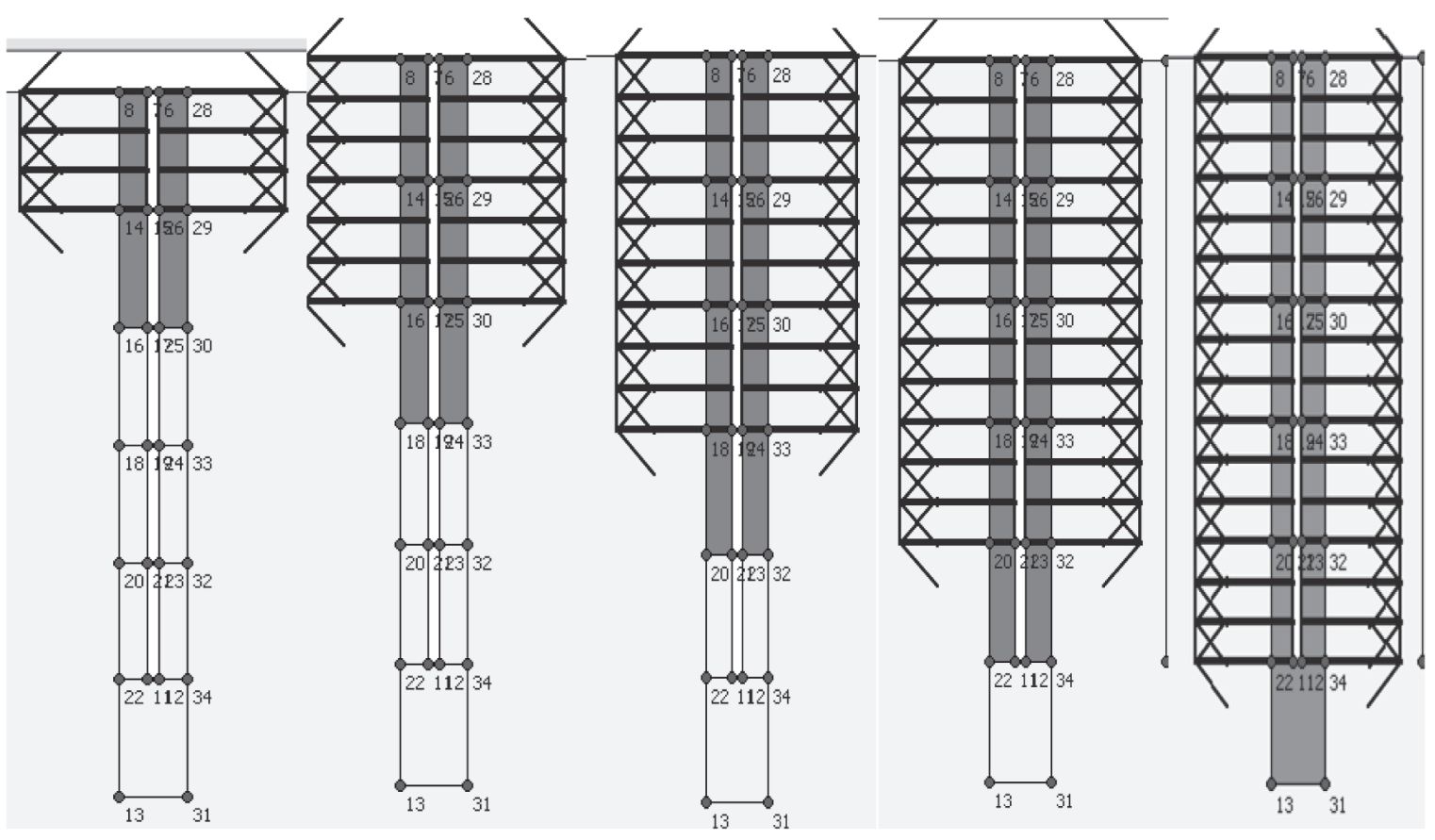

Figure 3 - Progressive expansion of cavity in five stages 


\section{Results}

In stiff clays, it was observed that the lateral displacement at the head of the adjacent pile becomes insensitive to Young's modulus of the soil, after a spacing of about $3.5 \mathrm{~d}$. However such trend cannot be observed in soft to medium clays, where the lateral displacement is sensitivity to the Young's modulus even at a spacing of $7.5 \mathrm{~d}$.
Figure 5 presents the numerical results for the lateral displacement of the head of the adjacent pile against the Young's modulus of the soil in a normalised form. The chart could be used to predict the lateral displacements of the head of the adjacent pile caused by pile driving in different soils.

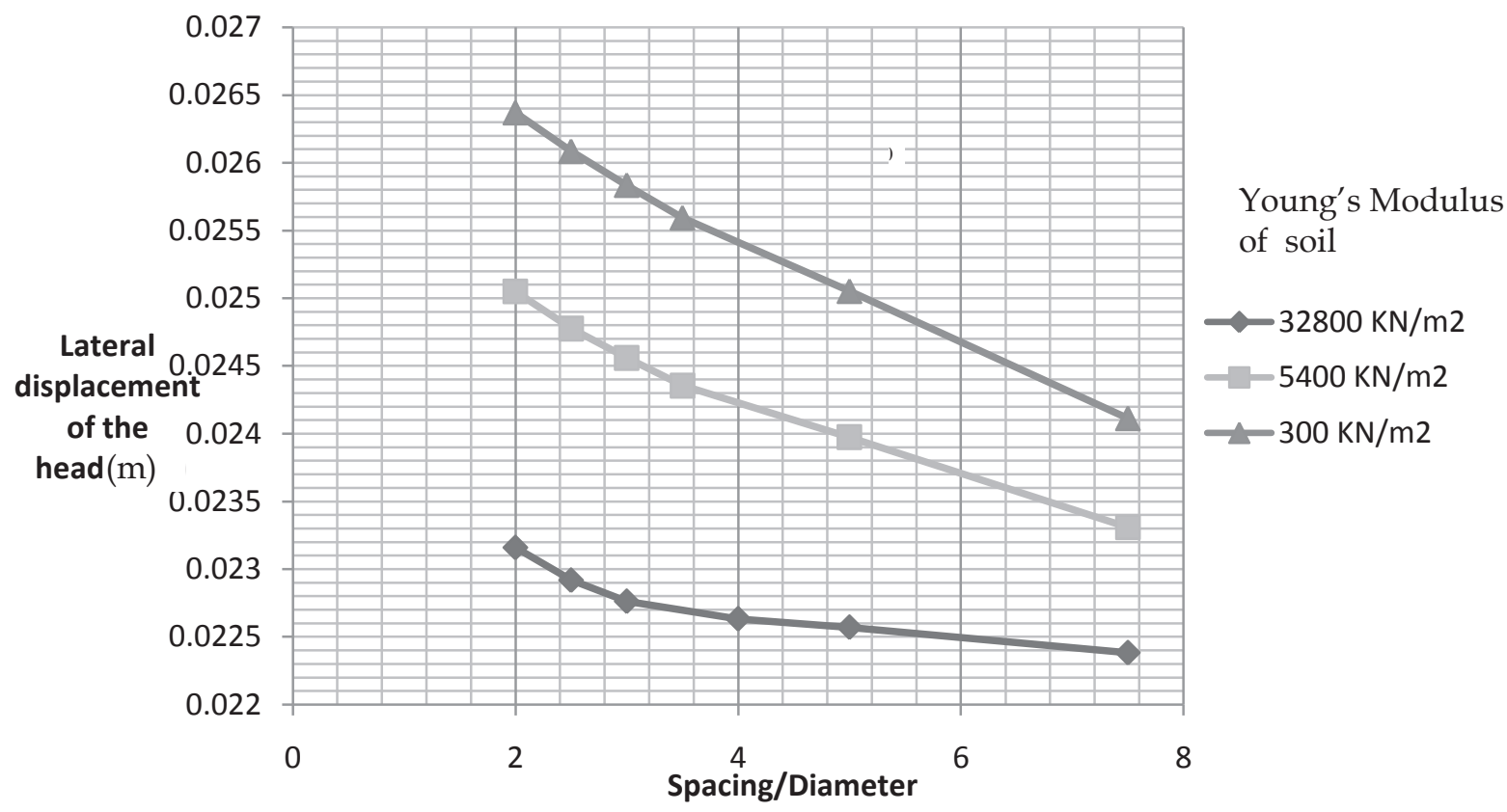

Figure 4 - Variation of lateral displacement of the head of the adjacent pile with spacing

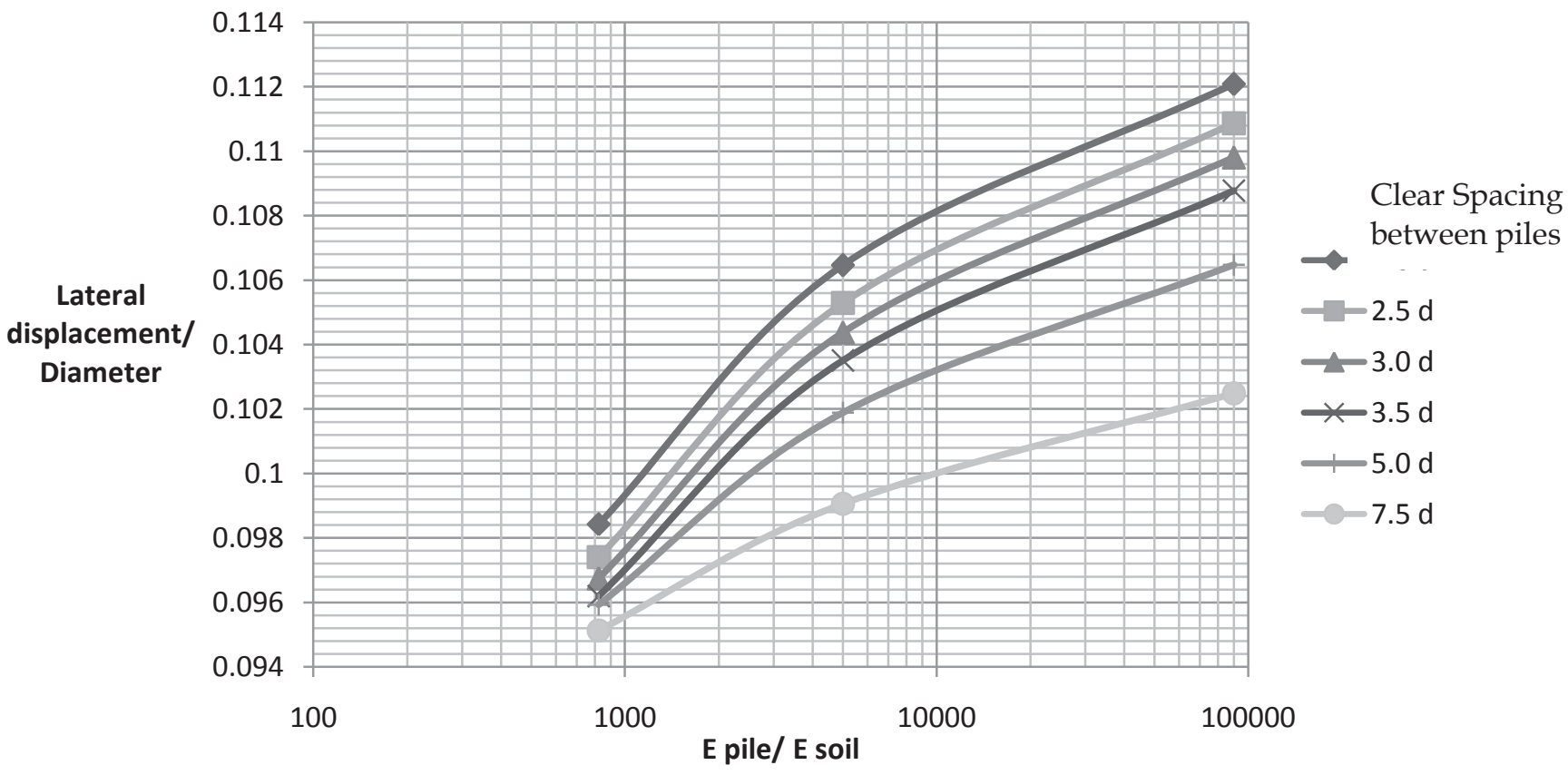

Figure 5 - Variation of lateral displacement of the head of the adjacent pile 
The results presented here are obtained for the smeared zone with Young's modulus of $100 \mathrm{kN} / \mathrm{m}^{2}$ and Poisson's ratio of 0.1 . These elastic properties were arbitrarily selected, therefore the analysis was repeated with different Young's moduli and Poisson's ratios of the smeared zone material. Results showed minor variations in the lateral displacement of the head of the adjacent pile with the Poisson's ratio of the smeared zone, whereas the variation with the Young's modulus was considerable, which is presented in Figure 6. The results for the lateral displacement of the adjacent pile are normalised with respect to the results predicted for Young's modulus ratio of 100 and it is called the correction factor. It could be used to modify the predicted results to get the displacement for any other ratios. One should estimate the Young's modulus of the smeared zone based on experience or actual field data if available, and results could be modified accordingly.

\section{Discussion}

Predicted results, presented above were obtained for undrained clays, (i.e. Poisson's ratio was 0.49 ) and for concrete piles, keeping ratio of the depth of the layer to the length of the pile as two.
As illustrated in Figure 4, the interaction between piles is considerable in weak to medium stiff clays. But in hard clays, the interaction is considerably lesser and at the same time beyond a spacing of $3.5 \mathrm{~d}$, it becomes negligible.

From Figure 6, it could be concluded that the interaction between piles in very stiff soils are negligible.

Calculations showed the tilt of the pile calculated as a ratio of head displacement relative to the tip of the pile to its length was insignificant, the maximum tilts were in the range of 1 to 350 . This shows that only an overall lateral displacement of the pile occurs due to the installation effect of an adjacent pile rather than the tilting.

The results obtained could be used together with constructional tolerances given for tilting and out of position of the pile. In weak clays the tolerances may be made stringent considering the interaction between piles due to installation.

\section{Conclusions and suggestions}

This study investigated the interaction between idealised two-dimensional piles due to pile driving. The results were obtained

\section{Correction Factor}

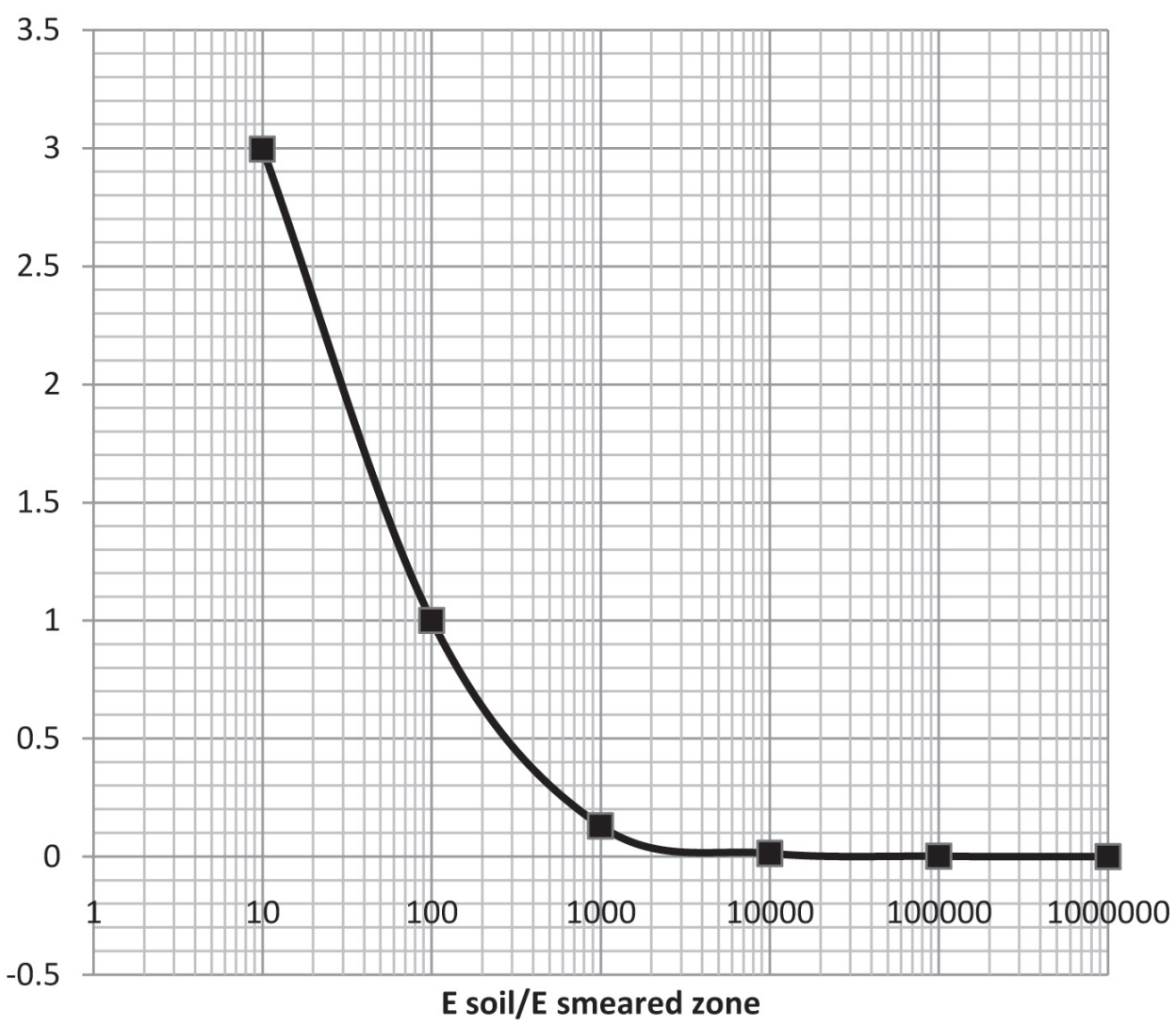

Figure 6 - Effect of smeared zone stiffness on the results 
numerically and tabulated, and could be used to predict the interaction of actual piling work to some extent. It was found that the lateral displacement of a nearby pile due to the driving of a pile decreases as the soil becomes stiffer, and has minimal effect for spacing in excess of 3.5 times the pile diameter. It is also shown that the driving action causes overall displacement of pile than the tiling if it is driven in weak soils. Results show that the constructional tolerances adopted for piling should be made more stringent in some cases

In future the results could be validated if any field data could be obtained. Furthermore variations of the results with different Poisson's ratios of the soil, different pile lengths, different pile diameters, different piling materials can be investigated and a modification factor could be derived to modify the presented results.

Further refinement of the predicted results could be achieved by conducting threedimensional finite element modelling, taking into account dynamic effects of pile driving, and using more complex constitutive models applicable to soils.

\section{References}

1. Terzaghi, K., Peck, R.B., Mesri, G., Soil Mechanics in Engineering Practice, 3rd ed., John Willey \& Sons, USA, 1996, p. 418.

2. Massout, M. E., and Tassoulas, J. O. L., A Finite Element Model for the Simulation of Pile Driving, International Journal for Numerical Methods in Engineering, 1994, Vol 37, pp 257-258.

3. Reference Manual - PLAXIS V8.2, Plaxis, Delft.

4. Poulus, G. H., Davis, E. H., Pile Foundation Analysis and Design, 1 1st ed, John Willey \& Sons, USA, 1980, p. 10.

5. Bearing Capacity of Soils, EM 110- 1- 1905, USACE, Dept. of the Army, Washington DC, 1983,p.5-45 \& 5-46

6. Bowles, J. E., Foundation Analysis and Design, $2^{\text {nd }}$ ed, McGraw-Hill, International, 1977. 
